\title{
Title: Evolution of carnivorous traps from planar leaves through simple shifts in gene expression
}

Authors: Christopher D Whitewoods ${ }^{1} \dagger$, Beatriz Gonçalves ${ }^{1} \dagger$, Jie Cheng ${ }^{1,2,3} \uparrow$, Minlong Cui ${ }^{4}$, Richard Kennaway ${ }^{1}$, Karen Lee ${ }^{1}$, Claire Bushell ${ }^{1}$, Man Yu${ }^{1}$, Chunlan Piao ${ }^{4}$ and Enrico Coen ${ }^{1 *}$

\section{Affiliations}

${ }^{1}$ Department of Cell and Developmental Biology, John Innes Centre, Norwich Research Park, Colney Lane, Norwich, NR4 7UH, UK.

${ }^{2}$ State Key Laboratory of Systematic and Evolutionary Botany, CAS Center for Excellence in

10 Molecular Plant Sciences, Institute of Botany, Chinese Academy of Sciences, Beijing, 100093, China.

${ }^{3}$ College of Life Sciences, University of Chinese Academy of Sciences, Beijing, 100039, China.

${ }^{4}$ College of Agriculture and Food Science, Zhejiang Agriculture and Forestry University, 15 Linan 311300, Zhejiang, China.

*Corresponding author: enrico.coen@jic.ac.uk

$\uparrow$ These authors contributed equally to this work.

Short title: Evolution of carnivorous traps from planar leaves 


\begin{abstract}
Leaves vary from planar sheets and needle-like structures, to elaborate cup-shaped traps. Here we show that in the carnivorous plant Utricularia gibba, the upper leaf (adaxial) domain is restricted to a small region of the primordium which gives rise to the trap's inner layer. This restriction is necessary for trap formation, as ectopic adaxial activity at early stages gives radialized leaves and no traps. We present a model that accounts for the formation of both planar and non-planar leaves through adaxialabaxial domains of gene activity establishing a polarity field that orients growth. In combination with an orthogonal proximodistal polarity field, this system can generate

30 diverse leaf forms, and can account for the multiple evolutionary origins of cup-shaped leaves through simple shifts in gene expression.
\end{abstract}

\title{
One Sentence Summary
}

A developmental model shows how shifts in gene activity can generate diverse leaf forms.

\section{Main Text}

Leaves come in many shapes and sizes. Most consist of planar sheets of cells that harvest light for photosynthesis. Formation of these leaves depends on adaxial and abaxial domains of gene activity in leaf primordia $(1,2)$. However, the mechanism by which these domains generate sheet-like development is unclear. It is unknown whether growth is oriented by the adaxial-abaxial (ad-ab) boundary throughout the leaf, or solely at the epidermis. It is also unclear how orientations of growth and cell division are specified; and whether growth orients the plane of division, or the plane of division orients growth. Finally, it is unclear how the system for planar leaf development has been modified to generate non-planar leaves, 
such as filiform (needle-like) leaves, and cup-shaped leaves of carnivorous plants which have evolved multiple times independently.

Computational models for formation of flat or cup-shaped leaves have been proposed based on cell divisions being induced by the epidermal ad-ab boundary, with the plane of division orienting growth $(3,4)$. However, these models are not easily reconciled with observations that cell divisions occur throughout the leaf lamina, not solely at the margin (5-7). Here we 50 suggest an alternative mechanism based on the analysis of ad-ab genes in trap and filiform leaf development of the humped bladderwort, Utricularia gibba (Lentibulariaceae), an aquatic carnivorous plant (Fig.1A).

Each $U$. gibba leaf consists of several filiform leaflets (Fig.1, E,F), and may bear a trap (Fig.1, D,F). At early developmental stages, organ primordia are dome-shaped (Fig. 1G).

55 Based on morphology alone, it is unclear at this stage whether these primordia will become leaflets or traps. At later stages, leaflet primordia form tapering cylinders that grow to be slightly wider than thick, and curve longitudinally towards the apex (Fig.1, H-J). By contrast, trap primordia are curved in both longitudinal and transverse sections, and consist of three cell layers (Fig.1K). The inner layer is positioned adaxially (facing the spiral apex, to the

60 right in Fig.1). As development progresses, the trap grows to a near-spherical shape with a closed mouth (Fig.1, L-O, white arrowheads), and a two-cell-thick trap door grows out near the dorsal lip (Fig.1, N-O, orange arrowheads). Over a 20-fold increase in trap length (approximately 400-fold increase in area), lamina thickness only doubles (Fig.1, K-O and Fig.S1), resulting in a curved sheet.

65 To define the ad-ab domains in $U . \operatorname{gibba}(8,9)$, we identified homologues of the adaxially expressed $P H V / P H B$ genes, and abaxially expressed $F I L$ and $K A N$ genes $(10,11)$. Before trap and leaflet primordia morphologies clearly diverged, $U g P H V 1$ was expressed on the 
adaxial side (black arrowhead Fig. 2A), and was more restricted in some primordia (yellow arrowhead Fig. 2A). In leaflet primordia at later stages, $U g P H V 1$ and $U g F I L 1$ were expressed on the adaxial and abaxial sides respectively (Fig.2, B-E; Fig.S3, A-J). In trap primordia $U g P H V 1$ was expressed in the innermost cell layer, extending into the inner side of the trap door (Fig.2, F-I; Fig.S3, K-P). UgFIL1 and $U g K A N 1$ were expressed in the outer layers (Fig. 2, J-Q, Fig.S3, Q-V), although only $U g K A N 1$ was expressed in the outer ventral region (yellow arrowhead Fig.2, N-Q). Thus, the adaxial and abaxial domains of a planar leaf broadly correspond to the inner and outer regions of the trap respectively. Similar findings were reported for $S$. purpurea trap, although $K A N$ expression was not detected (4).

To determine whether the observed expression patterns have functional significance, we induced ectopic expression of miRNA-resistant $U g P H V 1$ under the control of the $35 \mathrm{~S}$ promoter, using a Cre-Lox system ( $H S-U g P H V 1$ plants, see Methods for details). After 80 extended heat-shock, GFP fluorescence and in situ hybridization confirmed ectopic induction throughout the tissue (Fig.S4).

To determine how ectopic $U g P H V 1$ affected development, tissues were imaged daily following induction (Fig. 3, A-D). At seven days post-induction (Fig.3D), the main axis could be divided into three regions: (1) An upper region, encompassing the apex and leaves (Fig.3D, red). The normal spiral organization of the apex had been replaced by an open linear structure (Fig. 3, H-M), containing no trap primordia, and only radially symmetrical leaflets (Fig.S6). The leaves below the apex bore no traps, or bore small malformed traps (Fig.3D; Fig.S5, C-E). Tracing this upper region back through the sequence of daily images, showed it derived from primordia located within the spiral apex at the time of induction (Fig.3C;

90 Fig.S5B). (2) A middle region (Fig.3D, blue), derived from leaves bearing small traps (80 to $200 \mu \mathrm{m}$ long) at the time of induction (Fig.3D, white arrows, Fig.S5B). This region had normal leaves bearing traps up to $300 \mu \mathrm{m}$ long, with thick walls and malformed trap doors 
(Fig.S5, F-H). (3) A lower region, derived from leaves bearing traps that were longer than $200 \mu \mathrm{m}$ at the time of induction (Fig.3D, black; Fig.S5B). This region contained normal

95 leaves and traps.

Thus, ectopic expression of $U g P H V 1$ in early primordia leads to loss of trap development (Fig. 3, E,F), and generation of radialized organs, similar to the effect of ectopic $P H V / P H B$ in Arabidopsis (11-13). Ectopic UgPHV1 in later trap primordia, but before traps are $200 \mu \mathrm{m}$ long, leads to aberrant trap development and growth arrest (Fig.3G; Fig. S5). Ectopic $100 U g P H V 1$ after this stage has no effect, although this may be due to inefficiency of induction in older traps (Fig. S4). These results indicate that restricted $U g P H V 1$ is necessary for initiation and maintenance of trap development.

To explore how domains of ad-ab identity may control leaf morphogenesis, we modelled primordia as mechanically connected viscoelastic volumes of material, with growth oriented

105 by a polarity field (14). We began with a hemispherical ground state, representing a radialized leaf primordium without a history of ad-ab activity, with polarity pointing proximodistally (red arrows Fig. 4A). For simplicity, the field was implemented by taking the gradient of a morphogen diffusing from the base towards the tip. In reality, such fields most likely reflect cellular polarity rather than a continuous field $(15,16)$. Specifying a higher

110 growth rate parallel to, rather than perpendicular to, the polarity, generated a tapering cylinder, corresponding to the phenotype of radialized mutants (Fig.4, A-C; Movie S1).

To model formation of a planar leaf, we invoked a second polarity field, orthogonal to the first, termed the orthoplanar polarity field (black arrows Fig.4D). This field was implemented by taking the gradient of a morphogen diffusing from the outer surface of the primordium

115 towards the junction between adaxial and abaxial domains throughout the midplane. Evidence for a midplane domain playing a role in lamina formation comes from analysis of 
the WOX/PRS module (17-20). Three growth rates could then be specified: parallel to proximodistal polarity $\left(K_{p d}\right)$, parallel to the orthoplanar polarity $\left(K_{o p}\right)$ and perpendicular to both $\left(K_{p e r}\right)(14)($ Fig.4E).

120 Setting $K_{o p}$ to be much lower than $K_{p d}$ and $K_{p e r}$ generated a flattened sheet, corresponding to a planar leaf (Fig.4, F,G; Movie S2). Similar results were obtained using elliptical or elongated initial primordium shapes, illustrating the robustness of the model (Fig. S7). Low $K_{p e r}$ as well as $K_{o p}$ generated a tapering elliptic cylinder, corresponding to leaflet of $U$. gibba (Fig.4, H,I; Movie S3). Thus, the formation of a planar leaf can be accounted for by having relatively low $125 K_{o p}$, and a filiform leaf by both low $K_{o p}$ and low $K_{p e r}$.

To determine the effect of a more confined adaxial domain, as observed in some $U$. gibba primordia (Fig. 2A), we restricted the domain to a small region on one side of the primordium, while keeping the same values of $K_{p e r}, K_{o p}$ and $K_{p d}$ as in the planar leaf model (Fig.4, J,L). Running this model generated a cup-shaped sheet with the adaxial domain on the 130 inner surface (Fig.4, K,L; Movie S4). This result suggests that those primordia showing restricted PHVI expression at early stages (Fig. 2A) are fated to form traps. The model also predicts that expressing the adaxial domain throughout the primordium eliminates the orthoplanar polarity field, generating a tapering cylinder (Fig.4B), consistent with the results of ectopic PHV1 expression (Fig.3; Fig. S6).

135 In contrast to $U$. gibba, the adaxial domain in trap primordia of $S$. purpurea extends to the base of the primordium as a narrow ventral strip which grows out to form a ridge (4). Incorporation of such a strip within the above model led to the formation of a cup with a ridge, similar to the form observed in S. purpurea (Fig.4, M-P; Movie S5). In S. purpurea, cell divisions were observed to be preferentially periclinal (new cell walls parallel to the outer surface of the primordium) in the ridge-forming region, but not in the more distal cup- 
forming region (4). The authors incorporated these observations into a $2 \mathrm{D}$ model of a transverse section of the primordium, with small outgrowths prespecified at the ad-ab boundary. Assuming divisions are induced near the epidermal ad-ab boundary, and that the planes of division determine the orientation of growth, this model could generate the 145 observed 2D shapes (4).

To determine whether our model would give similar division patterns, we modelled transverse sections of the primordium containing virtual cells (Fig.4Q), with specified growth oriented relative to an orthoplanar polarity field. The plane of cell division was set by taking the shortest path through the cell center $(5,21)$. Some cells were marked in white to allow clones to be visualized. Running this model recapitulated the observed patterns of division (Fig.4, R,S; Movies S6, S7), with divisions in the ridge-forming region being preferentially periclinal (arrowed in Fig.4R). Thus, our model accounts for both trap morphogenesis and observed planes of division in S. purpurea.

Our findings provide a simple mechanistic explanation for the generation of planar leaves,

155 filiform and cup-shaped leaves, through shifts in expression domains or their effects on growth. Unlike previous models, growth is oriented by a polarity field, anchored by ad-ab domains acting throughout the leaf (not just at the epidermal boundary), consistent with observed division patterns. The planes of division are a result (emergent property), rather than the cause, of oriented growth. Moreover, our model does not depend on the primordium 160 already having outgrowths in the regions that form the lamina, showing that it can break morphological symmetry rather than simply elaborating it.

Additional structures, such as petioles, can be generated by introducing further domains into the model (Fig.S8-S10; Movies S8-S10). Diverse shapes and patterns of dissection in the outline of planar leaves may also be generated through modulation of growth oriented by a 
165 proximodistal polarity field (22-25). Thus, a system in which regional identities modify growth rates oriented by two orthogonal polarity fields provides developmental flexibility, and can account for how cup-shaped forms evolved multiple times independently from species with planar leaves.

\section{$170 \quad$ References}

1. J. R. Mcconnell, M. K. Barton, Development. 125, 2935-2942 (1998).

2. R. Waites, A. Hudson, Development. 121, 2143-2154 (1995).

3. Y. Hayakawa, M. Tachikawa, A. Mochizuki, J. Theor. Biol. 404, 206-214 (2016).

4. K. Fukushima et al., Nat. Commun. 6, 6450 (2015).

175 5. S. Fox et al., PLoS Biol. 16, 1-48 (2018).

6. K. J. I. Lee et al., PLoS Biol., 1-42 (2019).

7. R. S. Poethig, I. M. Sussex, Planta. 165, 170-184 (1985).

8. E. Ibarra-Laclette et al., Nature. 498, 94-8 (2013).

9. T. Lan et al., Proc. Natl. Acad. Sci., 201702072 (2017).

180 10. J. R. McConnell, M. K. Barton, Development. 125, 2935-2942 (1998).

11. J. R. McConnell et al., Nature. 411, 709-713 (2001).

12. J. F. Emery et al., Curr. Biol. 13, 1768-1774 (2003).

13. K. R. Siegfried et al., 4128, 4117-4128 (1999).

14. R. Kennaway, E. Coen, Open Biol. 9, 190057 (2019). 
185 15. K. Abley et al., Development. 140, 2061-74 (2013).

16. C. Mansfield et al., Curr. Biol. 28, 2638-2646.e4 (2018).

17. H. Lin et al., Proc. Natl. Acad. Sci. U. S. A. 110, 366-71 (2013).

18. M. Nakata et al., Plant Cell. 24, 519-35 (2012).

19. M. Vandenbussche et al., Plant Cell. 21, 2269-83 (2009).

190 20. M. J. Scanlon, R. G. Schneeberger, M. Freeling, Development. 122, 1683-1691 (1996).

21. L. Errera, CR Acad Sci. 103, 822-4 (1886).

22. T. Blein et al., Science. 322, 1835-9 (2008).

23. A. Hay, M. Tsiantis, Nat. Genet. 38, 942-7 (2006).

195 24. E. Kuchen et al., Science (80-. ). 335, 1092-1096 (2012).

25. A. Richardson, A. B. Rebocho, E. Coen, Plant Cell. 28, 2079-2096 (2016).

26. C. D. Whitewoods, E. Coen, Curr. Biol. 27, R910-R918 (2017).

27. K. Tamura, G. Stecher, D. Peterson, A. Filipski, S. Kumar, Mol. Biol. Evol. 30, 2725-9 (2013).

200 28. E. Aronesty, ea-utils : "Command-line tools for processing biological sequencing data." https://github.com/ExpressionAnalysis/ea-utils (2011).

29. C. Trapnell, L. Pachter, S. L. Salzberg, Bioinformatics. 25, 1105-1111 (2009).

30. A. Roberts et al., Genome Biol. 12, R22 (2011). 
31. C. Trapnell et al., Nat. Biotechnol. 31, 46-53 (2012).

205 32. A. B. Rebocho, J. R. Kennaway, J. A. Bangham, E. Coen, Curr. Biol. 27, 26102622.e3 (2017).

33. E. Weber, C. Engler, R. Gruetzner, S. Werner, S. Marillonnet, PLoS One. 6 (2011), doi:10.1371/journal.pone.0016765.

34. E. Truernit et al., Plant Cell. 20, 1494-503 (2008).

210 35. D. J. Cosgrove, Plant Physiol. 176, 16-27 (2018).

\section{Acknowledgements}

This work was supported by European Research Council grant (323028-CarnoMorph) and Biotechnology and Biological Sciences Research Council grants (BBS/E/J/000PR9787, BB/M023117/1, BB/L008920/1) awarded to EC. CB was funded by grant 1243441 from the

215 Biotechnology and Biological Sciences Research Council. BG was supported by a LongTerm Fellowship from the Federation of European Biochemical Societies. JC. was supported by the University of Chinese Academy of Sciences Joint PhD Training Program and the National Natural Science Foundation of China Grants (91631308). CDW and BG contributed biological experiments, data analysis and conceptualization, JC computational

220 modelling, data analysis and conceptualization, MC and CP plant transformation, RK software development and computational modelling, KL development of $U$. gibba resources, CB RNAseq and data analysis, MY biological experiments and data analysis, and EC supervision, funding acquisition and conceptualization.Authors have no competing interests. All data is available in the main text or the supplementary materials. Code is available at the following websites: https://github.com/JIC-Enrico-Coen/GFtbox-CarnivorousTraps-2019 and http://cmpdartsvr3.cmp.uea.ac.uk/wiki/BanghamLab/index.php/Software. 


\section{Supplementary Materials}

Materials and Methods

230 Figs. S1-S10

Tables S1-S6

Movies S1-S10

Data S1 
235 Figures and Legends

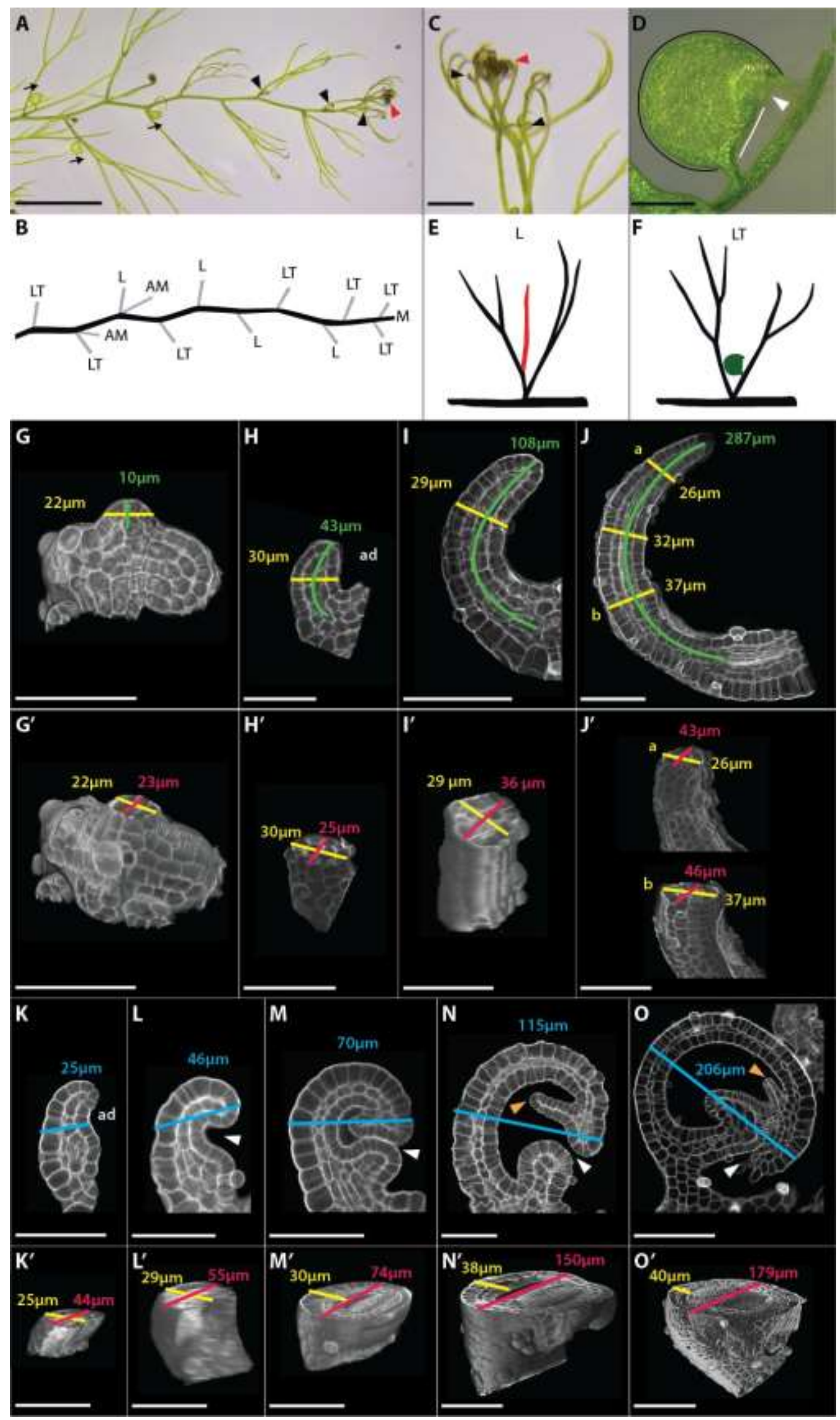


Fig. 1. Utricularia gibba morphology. (A) Stolon with circinnate apex (red arrowhead) bearing leaves and traps (black arrowheads) oriented adaxially (black arrows). (B) Schematic of (A) showing stolons (black) and leaves (grey). L leaflet-bearing leaf, $L T$ trap-bearing leaf, $240 A M$ axillary meristem, $M$ apical meristem. (C) Magnification of apex shown in (A). (D) Trap showing dorsal (black) and ventral (white) sides and mouth (arrowhead). (E) Diagram of $L$ in (B), with one leaflet in red. (F) Diagram of $L T$ in (B), with trap in green. ( $\mathbf{G}$ to $\mathbf{G}^{\prime}$ ) Longitudinal $(G)$ and oblique views of early primordium (G'). (H to J') Developmental series of leaflets in longitudinal section (H-J) and oblique view (H'-J'). (K to $\left.\mathbf{O}^{\prime}\right)$

245 Developmental series of traps in longitudinal section (K-O) and oblique view (K'-O'). In GO', green lines mark primordium and leaf length, yellow lines mark primordium, leaf and trap thickness, red lines mark primordium, leaf and trap width, blue lines mark trap length. Measurements are given in the same colors as the lines (see Fig. S1 for additional data). In NO, orange arrowhead marks trap door, white arrowhead marks trap mouth. In D, H-O' organs 250 are shown with adaxial side to the right, marked 'ad' on $\mathrm{H}$ and K. Scale bars, $5 \mathrm{~mm}$ (A), $1 \mathrm{~mm}(\mathrm{C}), 500 \mu \mathrm{m}$ (D), $50 \mu \mathrm{m}\left(\mathrm{G}, \mathrm{G}^{\prime}, \mathrm{H}, \mathrm{H}^{\prime}, \mathrm{I}_{\mathrm{I}} \mathrm{I}^{\prime}, \mathrm{J}^{\prime}, \mathrm{K}-\mathrm{N}^{\prime}\right), 100 \mu \mathrm{m}\left(\mathrm{J}, \mathrm{O}, \mathrm{O}^{\prime}\right)$. The image in N was previously published in (26). 

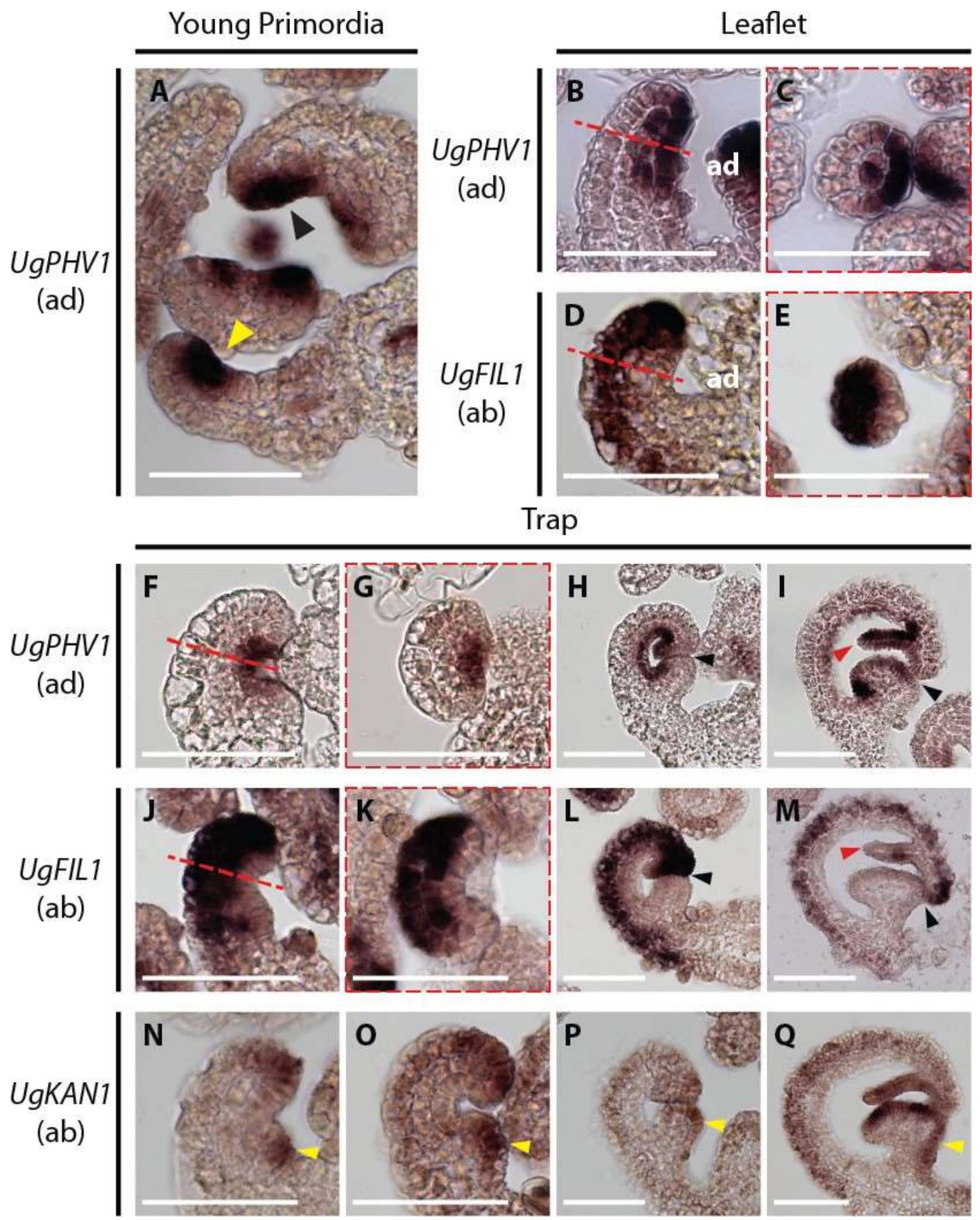

Fig. 2. UgPHVI and UgFILI expression patterns in Utricularia gibba leaflet and trap

development. (A) $U g P H V 1$ expression in young primordia. Black and yellow arrowheads mark primordia showing extended and restricted domains of the adaxial marker, respectively. 
(B to E) $U g P H V 1(\mathrm{~B}, \mathrm{C})$ and $U g F I L 1(\mathrm{D}, \mathrm{E})$ in leaflet primordia. (F to I) $U g P H V 1$ during trap 260 development, in longitudinal (F,H, I) and cross (G) sections. (J to M) UgFILI expression during trap development, in longitudinal (J,L,M) and cross (K) sections. (N to Q) UgKAN1 expression during trap development in longitudinal sections. Dashed red lines in E and $\mathrm{K}$ indicate angle of sections shown in G and K. Trap mouth (black arrowhead), trap door (red arrowhead), ventral region (yellow arrowhead). In all panels adaxial side is shown to the 265 right, marked 'ad' in B and D. Scale bars, $50 \mu \mathrm{m}$. See an expanded version of this figure in Fig. S3. 


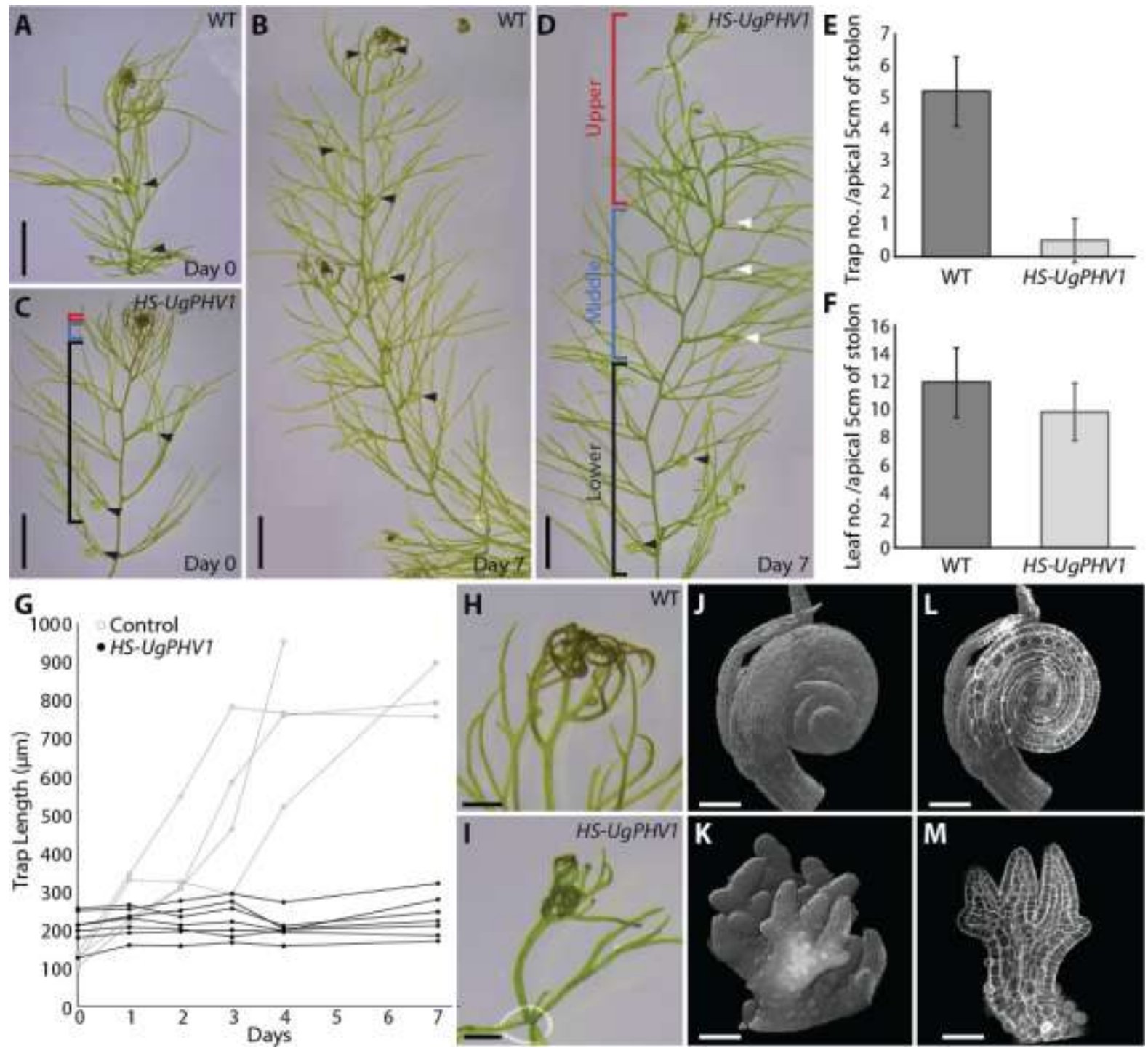

Fig. 3. Induction of $U \boldsymbol{g P H V} 1$ prevents trap formation. (A-F) Uninduced plants produce

traps (black arrowheads) at regular intervals $(\mathrm{A}, \mathrm{B})$, whereas induction of $U g P H V 1$ prevented new trap formation (C,D,E) and trap growth (white arrowheads), but not leaf formation (F). (G) Upon induction, traps smaller than $200 \mu \mathrm{m}$ did not grow. (H-M) Wild type circinnate apices $(\mathrm{H}, \mathrm{J}, \mathrm{I})$ and open apices of $H S-U g P H V 1$ plants with straight leaves (I,K,M). Scale bars in A to D $5 \mathrm{~mm}$; H and I $1 \mathrm{~mm}$; J to $\mathrm{M} 100 \mu \mathrm{m}$; L and M $50 \mu \mathrm{m}$. 
A
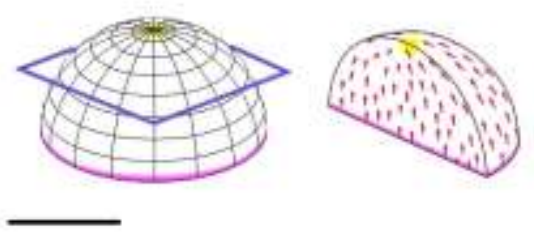

B

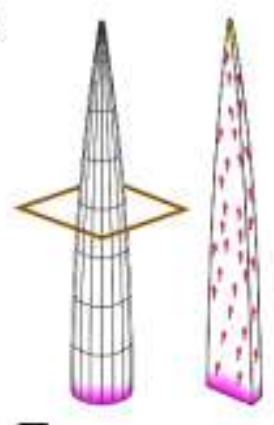

C
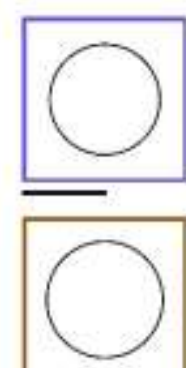

-

H

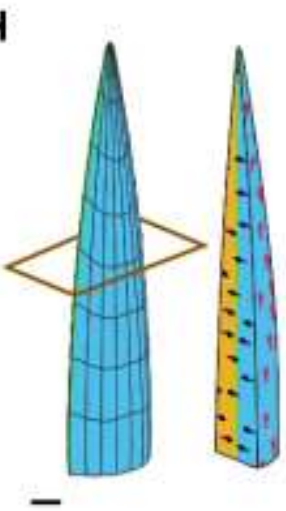

I
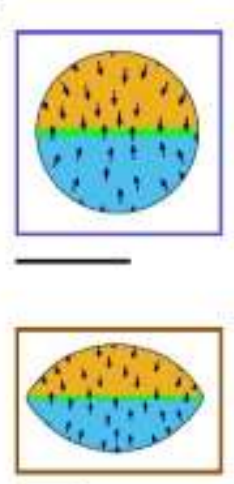

M

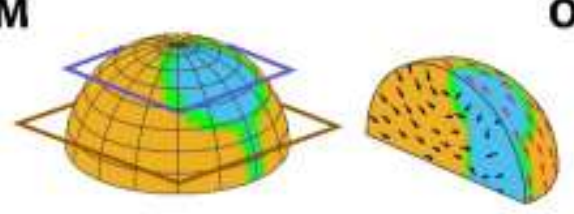

N
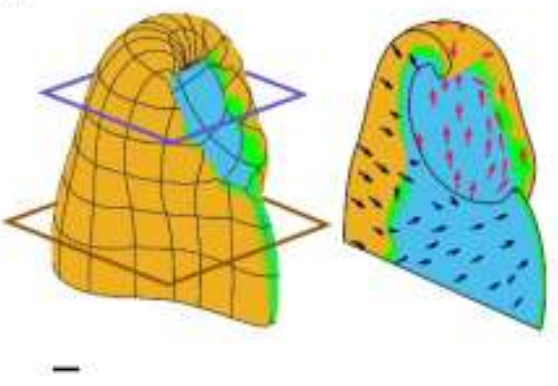

D
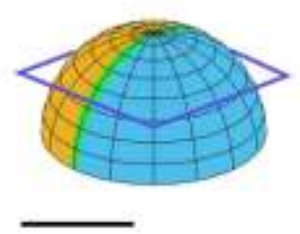

F
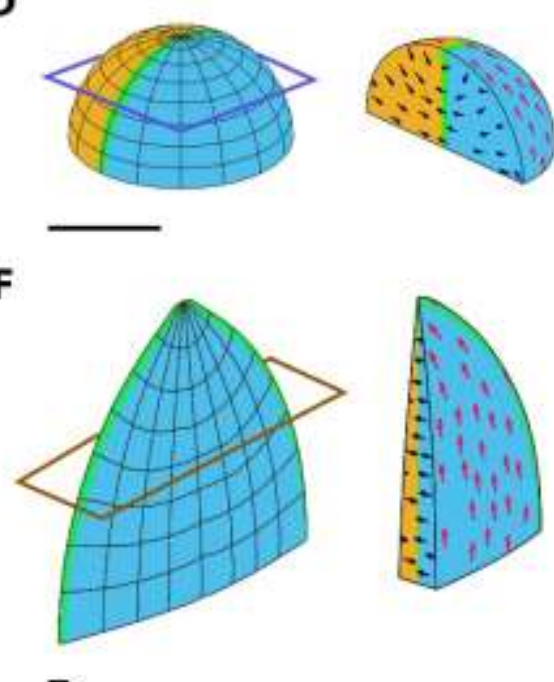

J
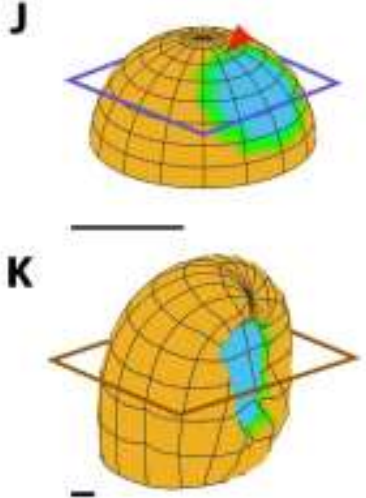

0
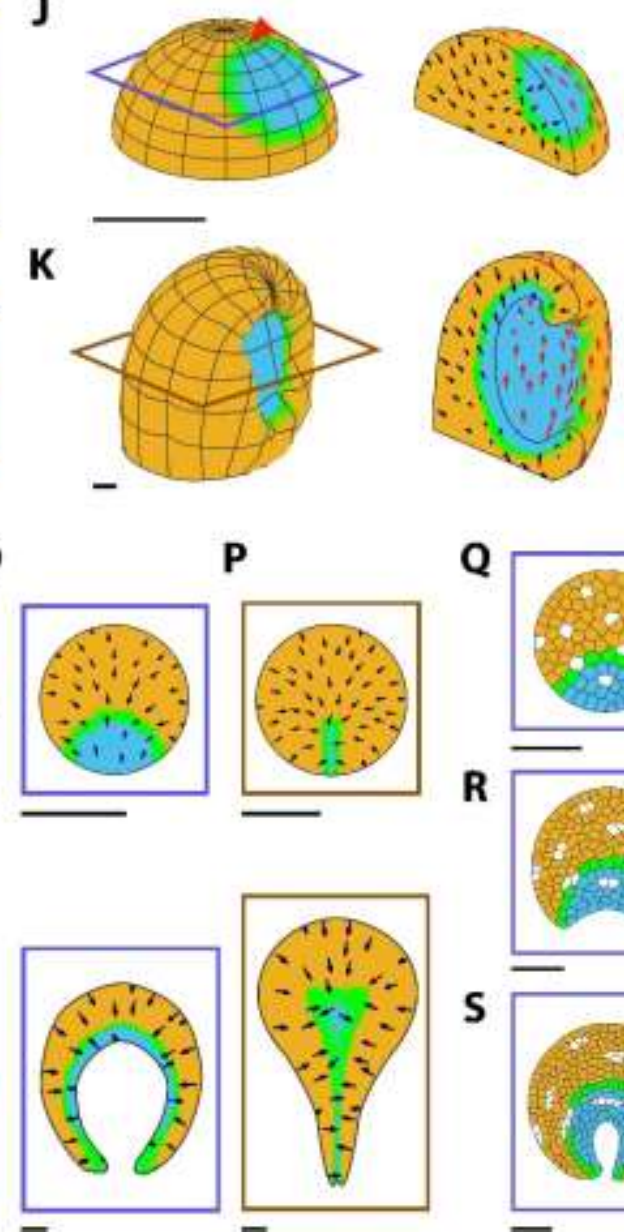

G

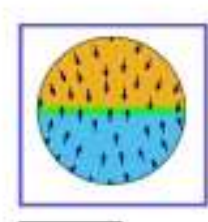

L
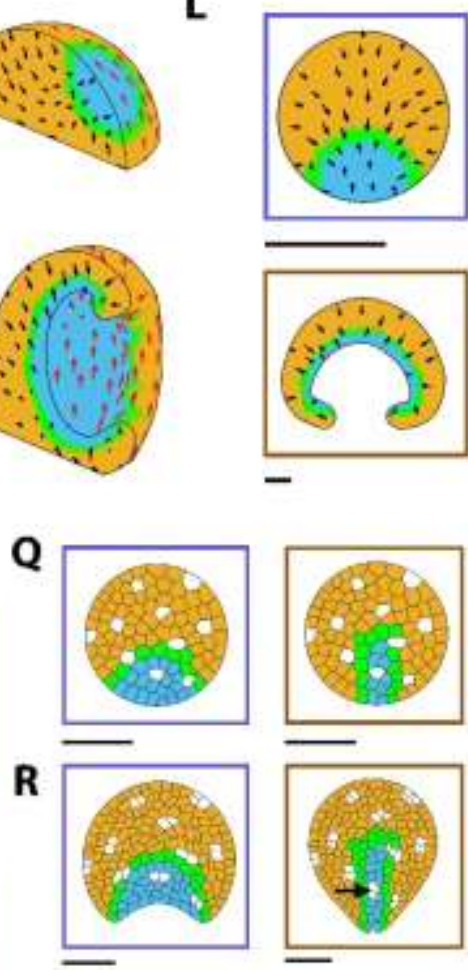

E
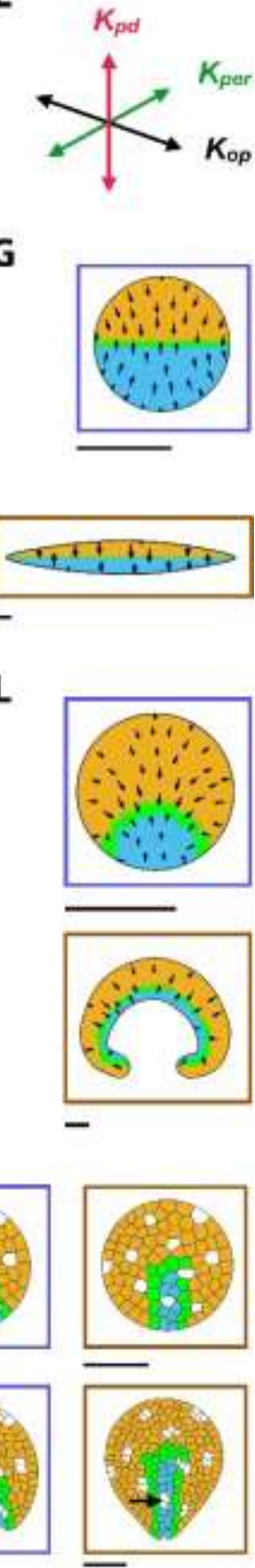

S

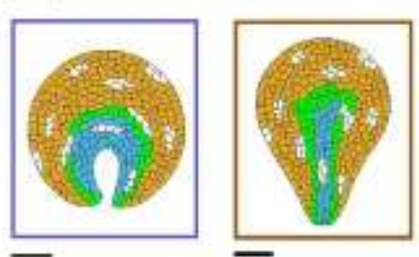

Fig. 4. Modelling of leaf and trap development. Oblique, cutaway and section views. (A-

C) Generation of tapering cylinder. Initial $(A, C)$ and final $(B, C)$ states. Proximodistal polarity

(red arrows) runs from organizers at base (magenta) to tip (yellow). (D-G) Generation of flat sheet. Initial $(\mathrm{D}, \mathrm{G})$ and final $(\mathrm{F}, \mathrm{G})$ states. Adaxial (blue), abaxial (brown) and midplane 
(green) domains. Orthoplanar polarity (black arrows) runs from surface to midplane.

Proximodistal and orthoplanar polarity shown only on outer and cutaway surfaces, respectively. Three growth rates specified by two polarity fields, $K_{p d}, K_{o p}$ and $K_{p e r}(\mathrm{E})$. (H and

285 I) Generation of an elliptic cylinder. Only final state shown. (J-L) Generation of a cup. Red arrowhead indicates shifted position of sink for proximodistal polarity. Initial (J,L) and final $(\mathrm{K}, \mathrm{L})$ states. (M-P) Generation of cup with ridge. Initial (M,O,P) and final (N,O,P) states. (Q-S) 2D models showing cell division patterns corresponding to section levels shown in $(\mathrm{M}, \mathrm{N})$. Initial (Q), intermediate (R) and final (S) states. Periclinal division walls in ridge 290 arrowed in (R). Levels of transverse sections indicated by color-coded rectangles. Scale bar in arbitrary units. 